\title{
Catalizadores tipo zinc(salen) y su aplicación en la copolimerización entre óxido de limoneno y $\mathrm{CO}_{2}$

\author{
Zinc(salen) catalysts and its application in the copolymerization of limonene oxide \\ and $\mathrm{CO}_{2}$
}

\author{
S. C. Acosta- Beltrán iD, C. E. Daza iD , C. Blanco-Jiménez iD
}

\begin{abstract}
Resumen- En este trabajo se lleva a cabo la síntesis y caracterización de catalizadores tipo salen con $\mathrm{Zn}$, los cuales son obtenidos mediante reacción de condensación entre una 1,2diamina y 3,5-diterc-butilsalicilaldehído en presencia de acetato de zinc. Se obtuvieron rendimientos del $86 \%$ en la síntesis del catalizador Zn-salen-1, 60\% para Zn-salen-2 y $70 \%$ para $\mathrm{Zn}$ salen-3. Estos catalizadores fueron usados en la reacción de acoplamiento de óxido de limoneno con $\mathrm{CO}_{2}$. Los mejores resultados en términos de conversión (20\%) se evidenciaron cuando el sistema catalítico $\mathrm{Zn}$-salen-2/NBuI se evaluó usando 20 bar de $\mathrm{CO}_{2}, 40^{\circ} \mathrm{C}$ durante $24 \mathrm{~h}$. En todos los casos estudiados, la reacción demostró ser completamente selectiva hacia la formación del policarbonato de limoneno. No se encontró dependencia significativa de la presión de $\mathrm{CO}_{2}$ en la determinación de la actividad del sistema catalítico examinado.
\end{abstract}

Palabras clave - copolimerización, dióxido de carbono, epóxido, óxido de limoneno, policarbonato.

\begin{abstract}
In this work we performed the synthesis and characterization of salen-type catalysts with $\mathrm{Zn}$, which were obtained by condensation reaction between a 1,2-diamine and 3,5ditertbutylsalicylaldehyde in the presence of zinc acetate. We reported yields of $86 \%$ in the synthesis of catalyst $\mathrm{Zn}$-salen-1, $60 \%$ for $\mathrm{Zn}$-salen-2 and 70\% for $\mathrm{Zn}$-salen-3. These catalysts were tested in the coupling reaction between $\mathrm{CO}_{2}$ and limonene oxide. The best results in terms of conversion $(20 \%)$ were achieved when the catalytic system Zn-salen-2/NBuI was evaluated under 20 bar of $\mathrm{CO}_{2}, 40{ }^{\circ} \mathrm{C}$ for $24 \mathrm{~h}$. In all cases studied, the reaction was completely selective towards the poly(limonene) carbonate formation. There was no significant dependence of $\mathrm{CO}_{2}$ pressure on the determination of the activity of the catalytic system examined.
\end{abstract}

Index Terms - carbon dioxide, copolymerization, epóxido, limonene oxide polycarbonate.

Este manuscrito fue enviado el 4 de agosto de 2017 y aceptado el 21 de junio de 2019.

Este artículo fue financiado por el proyecto "Copolimerización de epóxidos quirales con dióxido de carbono usando catalizadores homogéneos tipo salen con metales de transición" Código 28103 de la DIB-Universidad Nacional de Colombia.

S. C. Acosta- Beltrán Química egresada de la Universidad Nacional de Colombia. (email: scacostab@unal.edu.co).

\section{INTRODUCCIÓN}

olombia ocupa la posición 40 entre los países que emiten mayor cantidad de $\mathrm{CO}_{2}$, registrando en el 2012 una producción de 128 Mton de $\mathrm{CO}_{2}$, y un aporte del $0,42 \%$ al total de emisiones mundiales [1]. De acuerdo al más reciente inventario nacional de Gases de Efecto Invernadero (GEI) realizado por el Instituto de Hidrología, Meteorología y Estudios Ambientales (IDEAM); en Colombia, las actividades que más emisiones de $\mathrm{CO}_{2}$ generan son la agricultura y la ganadería (43\%) y el sector energético (44\%). El resto de la producción se distribuye entre la incineración de desechos industriales, la tala y quema de árboles y los procesos industriales como la fabricación de cemento [1].

En tal contexto, existen retos científicos para el presente siglo que por un lado se enfocan en reducir los niveles de dióxido de carbono atmosférico para mitigar los efectos del cambio climático $\mathrm{y}$, por otra parte, paralelamente, en velar por el desarrollo de materias primas alternativas y sostenibles que permitan la formación de productos químicos y nuevos materiales ambientalmente más amigables con el planeta.

Una de las alternativas a este serio problema es el diseño de procesos que permitan la captura y la fijación de dióxido de carbono conduciendo a la formación de productos químicos de valor añadido. El uso del $\mathrm{CO}_{2}$ como materia prima renovable ha despertado recientemente gran atención en la comunidad científica y en el mundo industrial [2-5]. Además de la problemática ambiental mencionada, este interés está asociado a la baja toxicidad que tiene el $\mathrm{CO}_{2}$, a que no es inflamable, y a que es económico y abundante, lo que lo convierte en un reactivo sostenible $y$ atractivo para llevar a cabo transformaciones químicas hacia compuestos de mayor valor $[6,7]$.

C.E. Daza. Profesor Asociado del Departamento de Química de la Universidad Nacional de Colombia, Sede Bogotá. Carrera 30 No. 45-03, Bogotá, Colombia. (email: cedazav@unal.edu.co)

${ }^{3}$ C. Blanco- Jiménez. Profesora Asistente del Departamento de Química de la Universidad Nacional de Colombia, Sede Bogotá. Carrera 30 No. 45-03, Bogotá, Colombia (email: cblancoj@unal.edu.co) 
En la actualidad, la copolimerización entre epóxidos y dióxido de carbono en presencia de catalizadores homogéneos es una reacción prometedora para convertir $\mathrm{CO}_{2}$, ya que permite la formación de policarbonatos y/o carbonatos cíclicos $[2,6,8$ 11]. Ambos productos poseen relevancia industrial. En primer lugar, los policarbonatos son polímeros muy especiales debido a sus propiedades físicas tales como alta ductilidad, transparencia, alta resistencia al impacto y biodegradabilidad [12]. Por su parte, los carbonatos cíclicos se utilizan como monómeros para la homopolimerización de policarbonatos y se emplean como disolventes apróticos polares, aditivos o como plastificantes [7, 13, 14]. Desde el punto de vista de la química verde, la producción de estos compuestos químicos es sostenible y puede conllevar a reducir las preocupaciones medioambientales considerando la posible disminución del $\mathrm{CO}_{2}$ en la atmósfera $[2,6]$.

Las investigaciones en la reacción de copolimerización con dióxido de carbono se han centrado en un número limitado de epóxidos, principalmente óxido de ciclohexeno [15-19], óxido de propileno [20-24] y óxido de estireno [23, 25-29]. Otros sustratos como el óxido de limoneno han comenzado a despertar interés [30-33] dado que al provenir de una fuente natural como el limoneno extraído de los frutos cítricos, pueden formar polímeros biodegradables en la reacción de copolimerización con $\mathrm{CO}_{2}[34,35]$. Lo anterior representa una ruta verde basada en materias primas no petroquímicas hacia la obtención de nuevos materiales.

A pesar del potencial de este sustrato, son escasos los estudios reportados en la literatura. En 2004, Coates et al. [30], reportaron la copolimerización alternada de óxido de limoneno y $\mathrm{CO}_{2}$ usando complejos de $\mathrm{Zn}$ - $\beta$-diiminatos [ $\left.\mathrm{ZnOAc}(\mathrm{BDI})\right]$. Los mejores resultados fueron obtenidos empleando una presión de 7 bar de $\mathrm{CO}_{2}, 25^{\circ} \mathrm{C}$ durante $24 \mathrm{~h}$. Estos autores encontraron una selectividad del $98,3 \%$ hacia la obtención de un policarbonato biodegradable con alto peso molecular (25 $\mathrm{kg} / \mathrm{mol}$ ).

Kleij et al. [33], investigaron la copolimerización de óxido de limoneno con $\mathrm{CO}_{2}$, utilizando como catalizador un sistema binario compuesto por un complejo de aminotrifenolato de $\mathrm{Al}(\mathrm{III})$ y PPNCl (PPN = bis(trifenil)fosfinoamonio). Los autores observaron $100 \%$ de selectividad hacia un policarbonato estereorregular de bajo peso molecular $(5,9$ $\mathrm{kg} / \mathrm{mol}$ ) con una conversión de $71 \%$ cuando la reacción se realizó en condiciones de 10 bar de $\mathrm{CO}_{2}$ y $42{ }^{\circ} \mathrm{C}$ durante $24 \mathrm{~h}$.

Recientemente, Kleij et al. [32], estudiaron la terpolimerización entre óxido de ciclohexeno y cis-óxido de limoneno con $\mathrm{CO}_{2}$ empleando un complejo de aminotrifenolato de $\mathrm{Al}(\mathrm{III})$ y $\mathrm{PPNCl}$ como cocatalizador. Los autores realizaron experimentos catalíticos con 15 bar de presión de $\mathrm{CO}_{2}$ y $40{ }^{\circ} \mathrm{C}$ obteniendo una conversión de $75 \%$ y selectividad $>95 \%$ hacia el terpolímero con un peso molecular de $3,96 \mathrm{~kg} / \mathrm{mol}$ al utilizar proporciones 1:1 de los dos epóxidos.

Por otro lado, los complejos metálicos salen han sido extensamente estudiados en la literatura dada su alta reactividad, selectividad y actividad en diversos procesos catalíticos [36-38]. Por ejemplo, los sistemas Zn-salen han mostrado ser catalizadores activos muy eficientes en la reacción de acoplamiento de epóxidos con $\mathrm{CO}_{2}$ para formar carbonatos cíclicos y/o policarbonatos $[8,18,39,40]$. Estos complejos han resultado interesantes por su facilidad de síntesis, estabilidad, y principalmente por qué ofrecen una modulación de los efectos estéricos y electrónicos en su estructura convirtiéndolos en los sistemas catalíticos más versátiles para catalizar reacciones como el acoplamiento de epóxidos con $\mathrm{CO}_{2}$.

En el presente artículo, se muestran los resultados de la síntesis y caracterización de tres catalizadores tipo salen con zinc. Adicionalmente, se detallan los resultados de los ensayos catalíticos llevados a cabo para la reacción de copolimerización de $\mathrm{CO}_{2}$ con óxido de limoneno utilizando $\mathrm{NBuI}$ como cocatalizador y los diferentes catalizadores tipo salen con zinc sintetizados. En este estudio se evaluó el efecto que tiene la presión de $\mathrm{CO}_{2}$ sobre la actividad y la selectividad de la reacción.

\section{METODOLOGÍA}

\section{A. Materiales y reactivos}

El (+)-óxido de limoneno (mezcla cis/trans) marca ALDRICH ( $97 \%$ de pureza) se almacenó en atmósfera inerte de nitrógeno y se utilizó tal y como se recibió. El precursor metálico $\mathrm{Zn}(\mathrm{OAc})_{2} \cdot 2 \mathrm{H}_{2} \mathrm{O}$ y las diaminas: etilendiamina, 1,2diaminociclohexano y 4-cloro-o-fenilendiamina fueron empleados sin previa purificación. El diclorometano usado en las reacciones catalíticas se secó sobre $\mathrm{P}_{2} \mathrm{O}_{5}$ y posteriormente fue almacenado bajo atmósfera inerte de nitrógeno y se desoxigenó antes de ser usado. El $\mathrm{CO}_{2}(99,99 \%)$ se usó sin previa purificación. El 3,5-diterc-butilsalicilaldehído fue empleado como precursor para la síntesis de los catalizadores salen, este reactivo se sintetizó siguiendo un procedimiento publicado en la literatura [41].

Los espectros de Resonancia Magnética Nuclear (RMN) ${ }^{1} \mathrm{H}$ y ${ }^{13} \mathrm{C}$ fueron registrados en un espectrómetro Bruker Advance $400 \mathrm{MHz}$.

\section{B. Síntesis de catalizadores}

Los catalizadores salen fueron sintetizados siguiendo procedimientos ya reportados con ligeras modificaciones [42]. Una disolución compuesta por la respectiva diamina $(1,0$ $\mathrm{mmol})$, 3,5-diterc-butilsalicilaldehído $\quad(2,1 \mathrm{mmol})$, $\mathrm{Zn}(\mathrm{OAc})_{2} \cdot 2 \mathrm{H}_{2} \mathrm{O}(1,1 \mathrm{mmol})$ y $\mathrm{NEt}_{3}(2 \mathrm{~mL})$ en $\mathrm{MeOH}(40 \mathrm{~mL})$ se dejó en reflujo por $2 \mathrm{~h}$ con agitación constante. Al cabo de este tiempo, se retiró el calentamiento y se dejó en agitación por $16 \mathrm{~h}$ a temperatura ambiente. Después, el metanol se evaporó casi hasta sequedad en rotaevaporador y el residuo se lavó con agua destilada $(30 \mathrm{~mL})$. El precipitado formado se filtró al vacío y se secó sobre sílica gel. El producto fue caracterizado por $\mathrm{RMN}-{ }^{-1} \mathrm{H}$. 


\section{Procedimiento general para la reacción de acoplamiento de óxido de limoneno con $\mathrm{CO}_{2}$}

Los experimentos catalíticos a alta presión se llevaron a cabo en un reactor batch de $100 \mathrm{~mL}$ de diseño propio construido en acero inoxidable y equipado con instrumentos de medición de temperatura y presión (ver Fig. 1).

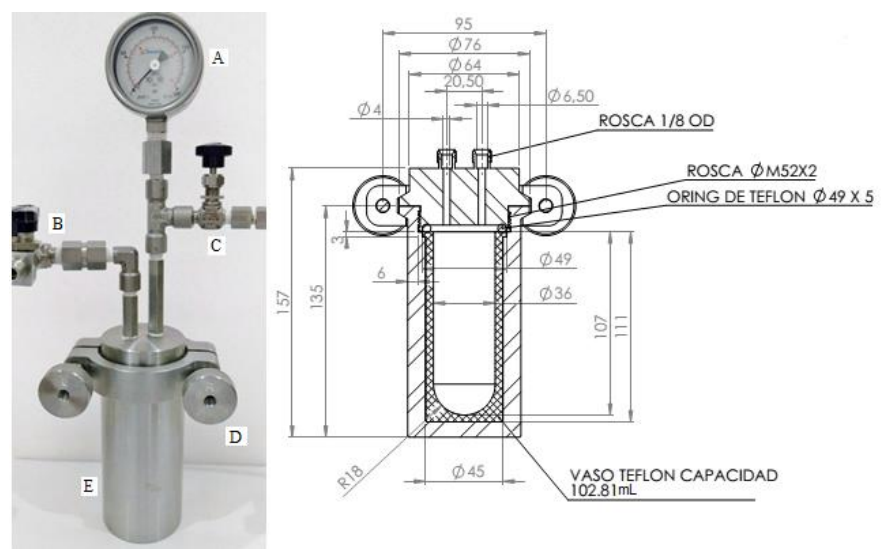

Fig. 1. Fotografía y esquema del reactor de alta presión, medidas en $\mathrm{mm}$. A. Manómetro, B. Válvula de ingreso de muestras, C. Válvula de presurización y venteo, D. Tornillos de seguridad, E. Vaso de reacción que contiene un recipiente de teflón interior.

En un experimento típico, una disolución del catalizador $(0,05$ $\mathrm{mmol}), \mathrm{NBuI}(0,05 \mathrm{mmol})$, y óxido de limoneno, $(2,1 \mathrm{~mL}, 12,5$ $\mathrm{mmol}$ ) en diclorometano seco $(5 \mathrm{~mL})$ se introdujo, por medio de una jeringa, dentro del reactor previamente conectado al vacío. Posteriormente, se presurizó el reactor con $\mathrm{CO}_{2}$ y el sistema se calentó a $40{ }^{\circ} \mathrm{C}$. Una vez alcanzado el equilibro térmico, se comenzó la agitación magnética. Después de $24 \mathrm{~h}$ de reacción, el reactor se enfrío a temperatura ambiente, y se liberó la presión del gas. La disolución resultante se filtró sobre celite, y al filtrado se le adicionó metanol, con lo cual se formó un precipitado blanco. Este sólido se aisló, secó y se caracterizó por $\mathrm{RMN}-{ }^{1} \mathrm{H}$ y $\mathrm{RMN}-{ }^{13} \mathrm{C}$.

\section{Determinación del porcentaje de conversión}

El porcentaje de conversión se calculó por $\mathrm{RMN}-{ }^{1} \mathrm{H}$ teniendo en cuenta la integración de señales características tanto para el sustrato como para el producto. Específicamente, para todos los crudos de reacción obtenidos en los ensayos catalíticos, se hizo seguimiento por $\mathrm{RMN}-{ }^{1} \mathrm{H}$ a la señal del protón metínico del grupo epóxido (Fig. 2, protón-G) que aparece en 2,98 ppm, así como a la señal resultante para el protón metínico hacia 5,01 ppm característica del policarbonato de limoneno protón-G' (Fig. 2).

Es importante resaltar que los espectros $\mathrm{RMN}-{ }^{1} \mathrm{H}$ son muy similares por lo cual es difícil realizar estos cálculos con señales distintas de las mencionadas. La presencia del policarbonato de limoneno se ratificó por $\mathrm{RMN}-{ }^{13} \mathrm{C}$ en donde se determinó que la señal a 150,04 ppm corresponde al desplazamiento característico para el carbono carboxílico cuando el acoplamiento de $\mathrm{CO}_{2}$ produce el polímero, siendo prueba concluyente de la formación de éste [30, 31].

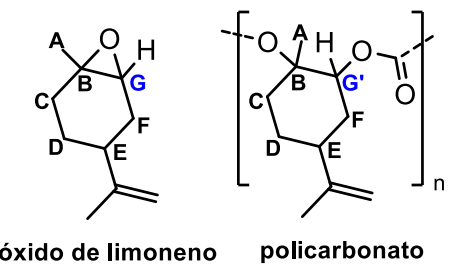

Fig. 2. Identificación de los átomos en el sustrato y en el producto principal para ser utilizadas en la descripción de las señales de RMN ${ }^{1} \mathrm{H}$.

\section{RESULTADOS Y DISCUSIÓN}

\section{A. Datos de RMN}

3,5-diterc-salicilaldehído: (60\% de rendimiento), RMN ${ }^{1} \mathrm{H}$ $\left(400 \mathrm{MHz}, \mathrm{CDCl}_{3}\right): \delta=1,33$ (s, 9H), 1,43 (s, 9H), 7,34, (d, 1H), 7,59 (d, 1H), 9,87 (s, 1H), 11,64 (s, 1H).

Complejo Zn-salen (Fig. 3, 1): (86\% de rendimiento), RMN ${ }^{1} \mathrm{H}$ (400MHz, Acetona-d $\mathrm{d}_{6}$ ): $\delta=1,27$ (s, 18H), 1,40 (s, 18H), 3,99 (s, $4 \mathrm{H}), 7,23(\mathrm{~d}, 2 \mathrm{H}), 7,38(\mathrm{~d}, 2 \mathrm{H}), 8,57$ (s, 2H).

Complejo Zn-salen (Fig. 3, 2): (60\% de rendimiento), RMN ${ }^{1} \mathrm{H}$ $\left(400 \mathrm{MHz}\right.$, Acetona- $\left.\mathrm{d}_{6}\right): \delta=1,16(\mathrm{~s}, 18 \mathrm{H}), 1,20(\mathrm{~s}, 18 \mathrm{H}), 7,19(\mathrm{~m}$, $2 \mathrm{H}), 1,23(\mathrm{~m}, 4 \mathrm{H}), 1,33(\mathrm{~m}, 4 \mathrm{H}), 1,37(\mathrm{~m}, 2 \mathrm{H}), 8,22(\mathrm{~s}, 1 \mathrm{H})$ y $8,28 \mathrm{ppm}(\mathrm{s}, 1 \mathrm{H})$.

Complejo Zn-salen (Fig. 3, 3): (70\% de rendimiento), RMN ${ }^{1} \mathrm{H}$ $\left(400 \mathrm{MHz}\right.$, Acetona- $\left.\mathrm{d}_{6}\right): 1,31(\mathrm{~s}, 18 \mathrm{H}), 1,53(\mathrm{~s}, 18 \mathrm{H}), 7,22(\mathrm{~m}$, $1 \mathrm{H}), 7,29(\mathrm{~m}, 1 \mathrm{H}), 7,34(\mathrm{~m}, 1 \mathrm{H}), 7,44(\mathrm{~m}, 2 \mathrm{H}), 7,91(\mathrm{~d}, 1 \mathrm{H})$, 7,94 (s, 1H), 9,06 (s, 1H), 9,09 (s, 1H).

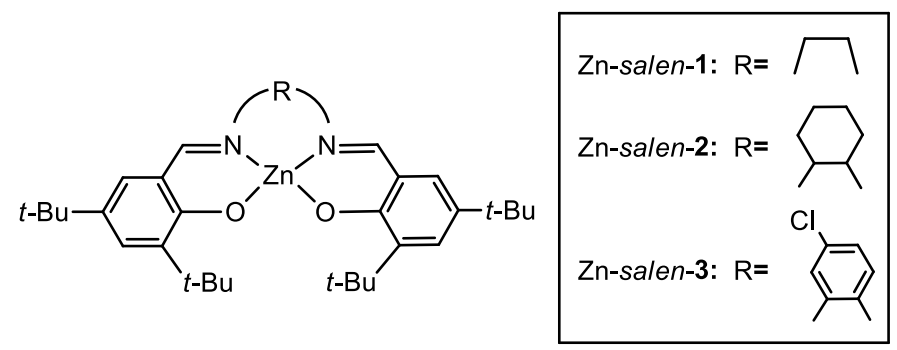

Fig. 3. Estructuras de los catalizadores tipo $\mathrm{Zn}$ (salen) 1-3

\section{B. Síntesis de catalizadores}

Los catalizadores sintetizados presentaron colores entre amarillo y naranja siendo solubles en la mayoría de disolventes orgánicos, estables al aire y a la humedad por lo que se manipularon y almacenaron en condiciones atmosféricas. Estos catalizadores $\mathrm{Zn}$-salen se seleccionaron en esta investigación debido a la facilidad de su síntesis, estabilidad, y alta actividad catalítica reportada [36-38] debido a sus propiedades como ácidos de Lewis, lo cual podría resultar conveniente en reacciones de acoplamiento de epóxidos con $\mathrm{CO}_{2}$.

Se obtuvieron rendimientos del $86 \%$ para el complejo $\mathrm{Zn}$-salen1, del 60\% para el complejo Zn-salen-2, y del $70 \%$ para el complejo Zn-salen-3 (Fig. 3). Dichos catalizadores fueron caracterizados por RMN ${ }^{1} \mathrm{H}$. Para el caso del complejo Zn- 
salen-1, el cual presenta incorporación de etilendiamina en el esqueleto diimina, el espectro de $\mathrm{RMN}{ }^{1} \mathrm{H}$, muestra un singulete a 1,27 ppm $(\mathrm{s}, 18 \mathrm{H})$ correspondiente a los grupos terc-butilos en posición para respecto al oxígeno del anillo aromático, y otro a $1,40 \mathrm{ppm}(\mathrm{s}, 18 \mathrm{H})$ de los grupos terc-butilos en posición orto; hacia 3,99 ppm $(\mathrm{s}, 4 \mathrm{H})$ aparece una señal asignada a los hidrógenos metilénicos del grupo 1,2-etanodiil, entre 7,23-7,38 (m, 4H) los hidrógenos del anillo aromático, y finalmente hacia $8,57 \mathrm{ppm}(\mathrm{s}, 2 \mathrm{H})$ una señal asignada a los hidrógenos del grupo imino.

Los espectros RMN ${ }^{1} \mathrm{H}$ de los complejos $\mathrm{Zn}$-salen-2 y Znsalen-3, presentaron igualmente las señales características de estas estructuras debido a la presencia de los dos grupos tercbutilsalicilaldehídos en la estructura del catalizador, como se describió anteriormente. Adicionalmente, para el complejo Znsalen-2, se observaron entre 1,23-1,37 ppm (m, 10H) las señales correspondientes al anillo cíclico por la incorporación del grupo 1,2-ciclohexanodiil. De otro lado, el complejo Zn-salen-3 presentó entre 7,34-7,94 (m, 3H) un multiplete correspondiente a los hidrógenos aromáticos del grupo 1,2-difeniil-4-cloro, que se explican dada la incorporación de 4-cloro-o-fenilendiamina en la estructura del catalizador.

Para todos los catalizadores se observó la desaparición de la señal en 11,64 ppm $(1 \mathrm{H})$ que se asigna al protón del grupo hidroxilo del 3,5-diterc-butilsalicilaldehído, confirmando la coordinación con el zinc. Así mismo, el protón del grupo aldehído del 3,5-diterc-butilsalicilaldehído se desplazó hacia campo alto debido al cambio de grupo funcional por la adición de la diamina formando una imina. Este desplazamiento se observó de 9,87 ppm (1H) a 8,57 ppm (1H) en el complejo $\mathrm{Zn}$ salen-1, de 9,87 ppm (1H) a 8,28 y $8,22 \mathrm{ppm}(1 \mathrm{H})$ en el $\mathrm{Zn}$ salen-2, y de 9,87 ppm (1H) a 9,09 ppm (1H) en el complejo $\mathrm{Zn}$-salen-3, después del cambio del grupo funcional.

\section{Copolimerización de óxido de limoneno con $\mathrm{CO}_{2}$ : Efecto de la presión}

Se estudió el efecto de la presión a 20,30 y 40 bar de $\mathrm{CO}_{2}$ en la reacción de copolimerización con óxido de limoneno (Fig. 4). Los resultados de los ensayos catalíticos se muestran en la Tabla I. En todos los casos (entradas 1-9) se encontró que los sistemas catalíticos binarios $\mathrm{Zn}$-salen/NBuI fueron quimioselectivos hacia el policarbonato de limoneno (\%PC>99). En ningún caso se evidenció la presencia del carbonato cíclico. Estas observaciones están en concordancia con la predominante selectividad hacia el policarbonato evaluada en la copolimerización del óxido de ciclohexeno con catalizadores similares tipo salen $[3,4,8,13,18]$.

Cuando el complejo Zn-salen-1 se empleó como catalizador, se observó que los valores de conversión a 20 y 30 bar de presión no presentan diferencias significativas (13 y $12 \%$ respectivamente) (Tabla I, entradas 2-3). Sin embargo, cuando la presión de $\mathrm{CO}_{2}$ se incrementó hasta 40 bar la conversión disminuyó hasta $8 \%$ (entrada, 3 ).

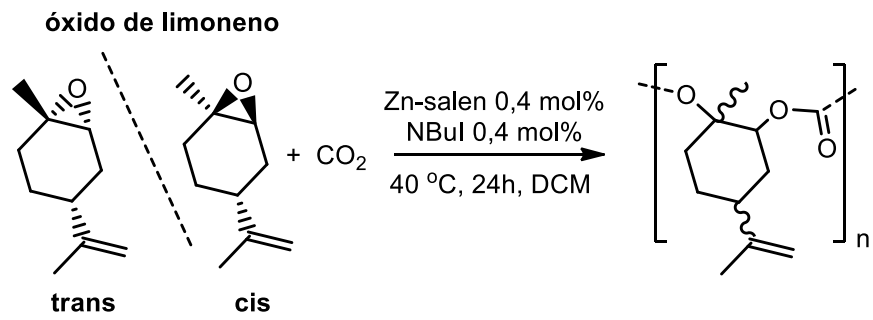

Fig. 4. Reacción de copolimerización de óxido de limoneno con $\mathrm{CO}_{2}$ en presencia de catalizadores tipo Zn-salen.

Por su parte, cuando se estudió la reacción catalítica en presencia del complejo Zn-salen-2 (Fig. 3) a 20 bar de presión de $\mathrm{CO}_{2}$, se obtuvo la mayor conversión (20\%) de todos los experimentos realizados (entrada 4). Sin embargo, se registró una disminución en la conversión (11\%) cuando la reacción se llevó a cabo a presiones de 30 y 40 bar (entradas 5 y 6) sin diferencias significativas entre los dos experimentos.

Estos resultados sugieren que con los catalizadores $\mathrm{Zn}$-salen-1 y -2 a altas concentraciones de $\mathrm{CO}_{2}$ puede ocurrir un posible efecto de dilución del sistema catalizador/sustrato provocando una disminución en la velocidad de reacción lo que redunda en un decrecimiento en la conversión. Este efecto de dilución fue reportado por Taherimehr et al. [40] para el sistema $\mathrm{Zn}($ salphen $) / \mathrm{NBuI}$ con diferentes tipos de epóxidos señalando que un incremento en la concentración de reactivos (presión de $\mathrm{CO}_{2}$ ) en presencia de disolvente provoca un deficiente contacto entre el catalizador, sustrato y $\mathrm{CO}_{2}$.

TABLA I

EFECTO DE LA PRESIÓN EN LA COPOLIMERIZACIÓN DE $\mathrm{CO}_{2}$ CON ÓXIDO DE LIMONENO $^{\mathrm{a}}$

\begin{tabular}{ccccc}
\hline \hline Entrada & Catalizador & $\mathrm{P} \mathrm{CO}_{2}($ bar $)$ & $\% \mathrm{C}^{\mathrm{b}}$ & $\% \mathrm{PC}^{\mathrm{b}}$ \\
\hline 1 & Zn-salen-1 & 20 & 13 & $>99$ \\
2 & Zn-salen-1 & 30 & 12 & $>99$ \\
3 & Zn-salen-1 & 40 & 8 & $>99$ \\
4 & Zn-salen-2 & 20 & 20 & $>99$ \\
5 & Zn-salen-2 & 30 & 11 & $>99$ \\
6 & Zn-salen-2 & 40 & 11 & $>99$ \\
7 & Zn-salen-3 & 20 & 7 & $>99$ \\
8 & Zn-salen-3 & 30 & 12 & $>99$ \\
9 & Zn-salen-3 & 40 & 12 & $>99$ \\
\hline \hline
\end{tabular}

${ }^{\mathrm{a}}$ Condiciones generales: $0,05 \mathrm{mmol}, 0,4 \mathrm{~mol} \%$ catalizador, $12,5 \mathrm{mmol}, 2,1$ $\mathrm{mL}$ sustrato, relación molar cat/cocat $/$ sust $=1 / 1 / 250,40^{\circ} \mathrm{C}, \mathrm{t}=24 \mathrm{~h}, \mathrm{DCM}(5$ $\mathrm{mL}$ ) como disolvente. ${ }^{\mathrm{b}} \% \mathrm{C}=$ Conversión, $\% \mathrm{PC}=$ Selectividad hacia el policarbonato. Determinadas por $\mathrm{RMN}{ }^{1} \mathrm{H}$ de las mezclas de reacción.

Finalmente, el catalizador Zn-salen-3 (Fig. 3) registró una conversión del 7\% (entrada 7) con 20 bar de presión. Se detectó un incremento en la conversión cuando la presión se elevó a 30 y 40 bar de $\mathrm{CO}_{2}$ (entradas 8 y 9) sin diferencias significativas entre los dos experimentos. Para este catalizador, el efecto de dilución no fue significativo.

Es importante mencionar que el uso del diclorometano (como disolvente) fue importante para obtener actividad catalítica, ya que favoreció la solubilidad del catalizador y cocatalizador en el medio de reacción. Al respecto, cuando las reacciones catalíticas se llevaron a cabo en ausencia de disolvente, no se observó ninguna conversión. A pesar de que en la literatura se reporta que el efecto de dilución es importante cuando se usa disolvente, se debe tener en cuenta que los experimentos fueron 
desarrollados bajo condiciones suaves de presión que no son suficientes para alcanzar el estado supercrítico del $\mathrm{CO}_{2}$ el cual puede mejorar la solubilidad entre reactivos. De igual manera, el uso del disolvente facilitó la operación del reactor pues no se hizo necesario el uso de caja seca para la manipulación de reactivos a diferencia de los experimentos reportados en la literatura [40].

Aunque las conversiones obtenidas fueron bajas comparadas con aquellas reportadas con otros catalizadores [30, 31], los resultados indicaron que el sistema catalítico es activo y selectivo hacia la formación del polímero del cual ya se mencionaron las posibles aplicaciones e interés ya que proviene de una fuente natural, sostenible como lo es el limoneno extraído de frutas cítricas.

Los resultados catalíticos reflejaron que la selectividad de la reacción no se ve afectada por las variaciones tanto estéricas como electrónicas en la estructura de los catalizadores ni por los cambios en la presión del gas. Sin embargo, se observó que el porcentaje de conversión del sustrato se modifica dependiendo del tipo de catalizador, siendo el catalizador $\mathrm{Zn}$ salen-2, aquel que presentó la mayor actividad bajo condiciones suaves de reacción entre los catalizadores estudiados.

Martín y Kleij, [43] estudiaron el sistema $\mathrm{Zn}($ salen $) / \mathrm{NBu}_{4} \mathrm{I}$ en la copolimerización de 1,2-epoxi-hexano reportando una ley de velocidad de primer orden respecto al cocatalizador y de primer orden respecto al catalizador, aunque no se explica la dependencia de la cinética respecto al $\mathrm{CO}_{2}$. Por otra parte, Moore et al. [44] reportaron que para catalizadores complejos $\mathrm{Zn}(\beta-\mathrm{BDI})$ usados en la copolimerización de óxido de ciclohexeno, el orden de reacción es cero respecto a $\mathrm{CO}_{2}$. Por analogía con estos sistemas, se podría esperar que, para la copolimerización de óxido de limoneno estudiada en este artículo, se siga una ley de velocidad similar en donde el orden de reacción del $\mathrm{CO}_{2}$ sea cero lo cual explicaría la no dependencia de los resultados respecto a la presión de $\mathrm{CO}_{2}$. Se fundamenta así que los resultados de conversión catalítica obtenidos son producto únicamente de un efecto de dilución y no de un control cinético, siendo importante el contacto efectivo entre reactivos.

Por lo general, los sistemas catalíticos tipo Zn-salen estudiados en esta investigación se han empleado para catalizar la formación de carbonatos cíclicos de epóxidos en presencia de $\mathrm{CO}_{2}[9,36,45,46]$. Los resultados obtenidos indicaron que la reacción entre el epóxido de limoneno y el $\mathrm{CO}_{2}$ catalizada por el sistema binario $\mathrm{Zn}$-salen/NBuI es selectiva a la formación del copolímero con una baja conversión en comparación con los resultados reportados en la literatura [30, 32, 33].

A partir de los resultados del presente trabajo se recomienda: llevar a cabo experimentos bajo condiciones supercríticas, estudiar otros parámetros de operación de la reacción con el fin de incrementar la conversión del sustrato, estudiar el efecto que tienen otros solventes para reemplazar el uso de diclorometano e incrementar la sostenibilidad del proceso. Por último, hacer un estudio cinético detallado de la reacción estudiada.

\section{CONCLUSIONES}

Se sintetizaron y caracterizaron tres catalizadores tipo salen con $\mathrm{Zn}$ con rendimientos del $86 \%$ para Zn-salen-1, del $60 \%$ para el complejo Zn-salen-2 y del 70\% para el complejo Zn-salen-3. La actividad catalítica de los complejos metálicos se evaluó en la reacción de acoplamiento de $\mathrm{CO}_{2}$ con óxido de limoneno. Se encontró que la presión no juega un papel determinante en la selectividad de la reacción. En todos los casos, la quimioselectividad fue completa hacia la formación del policarbonato de limoneno. Los mejores resultados en cuanto a conversión (20\%) se obtuvieron con el catalizador Zn-salen-2 a $40^{\circ} \mathrm{C}, \mathrm{t}=24$ h y 20 bar de presión.

Este trabajo plantea una ruta de transformación del $\mathrm{CO}_{2}$ hacia la formación selectiva de un material polimérico biodegradable a partir de un sustrato que proviene de una fuente natural y renovable mediante el uso de catalizadores fáciles de sintetizar, estables, con un metal de baja toxicidad, económico, factores que hacen sostenible al proceso estudiado.

\section{AGRADECIMIENTOS}

Los autores agradecen la financiación al proyecto "Copolimerización de epóxidos quirales con dióxido de carbono usando catalizadores homogéneos tipo salen con metales de transición" Código 28103 de la DIB-Universidad Nacional de Colombia.

\section{REFERENCIAS}

[1] A. D. Pulido, R. Jiménez, J. D. Turriago, and J. E. Mendoza, "Inventario Nacional de Gases de Efecto Invernadero (GEI) de Colombia," IDEAM, PNUD, MADS, DNP, CANCILLERIA, FMAM, Bogotá, Colombia, Jan. 2015.

[2] H. Büttner, L. Longwitz, J. Steinbauer, C. Wulf, and T. Werner, "Recent Developments in the Synthesis of Cyclic Carbonates from Epoxides and $\mathrm{CO}_{2}$," Topics in Current Chemistry, vol. 375, Mar. 2017. DOI: 10.1007/s41061-0170136-5.

[3] M. R. Kember, A. Buchard, and C. K. Williams, "Catalysts for $\mathrm{CO}_{2}$ /epoxide copolymerisation," Chemical Communications, vol. 47, pp. 141-163, Jan. 2011. DOI: 10.1039/C0CC02207A.

[4] X.-B. Lu, W.-M. Ren, and G.-P. Wu, "CO 2 Copolymers from Epoxides: Catalyst Activity, Product Selectivity, and Stereochemistry Control," Accounts of Chemical Research, vol. 45, pp. 1721-1735, Oct. 2012. DOI: 10.1021/ar300035z. Y. Qin, X. Sheng, S. Liu, G. Ren, X. Wang, and F. Wang, "Recent advances in carbon dioxide based copolymers," Journal of $\mathrm{CO}_{2}$ Utilization, vol. 11, pp. 3-9, Sept. 2015. DOI: 10.1016/j.jcou.2014.10.003.

[6] R.-R. Ang, L. Tin Sin, S.-T. Bee, T.-T. Tee, A. A. H. Kadhum, A. R. Rahmat, et al., "A review of copolymerization of green house gas carbon dioxide and oxiranes to produce polycarbonate," Journal of Cleaner Production, vol. 102, pp. 1-17, Sept. 2015. DOI: 10.1016/j.jclepro.2015.04.026. 
[7] M. Taherimehr and P. P. Pescarmona, "Green polycarbonates prepared by the copolymerization of $\mathrm{CO}_{2}$ with epoxides," Journal of Applied Polymer Science, vol. 131, pp. 1-17, Aug. 2014. DOI: 10.1002/app.41141.

[8] D. J. Darensbourg, "Making Plastics from Carbon Dioxide: Salen Metal Complexes as Catalysts for the Production of Polycarbonates from Epoxides and $\mathrm{CO}_{2}, "$ Chemical Reviews, vol. 107, pp. 2388-2410, Jun. 2007. DOI: $10.1021 / \mathrm{cr} 068363 \mathrm{q}$.

[9] J. E. Gómez and A. W. Kleij, "Recent progress in stereoselective synthesis of cyclic organic carbonates and beyond," Current Opinion in Green and Sustainable Chemistry, vol. 3, pp. 55-60, Feb. 2017. DOI: 10.1016/j.cogsc.2016.11.005.

[10] J. Wang, J. Mu, H. Su, S. Shan, Y. Wang, and Q. Jia, "Progress in the selectivity of reaction of carbon dioxide and epoxides," Gaofenzi Cailiao Kexue Yu Gongcheng/Polymeric Materials Science and Engineering, vol. 30, pp. 186-190, Feb. 2014. DOI: 10.1021/ma1021456.

[11] P. P. Pescarmona and M. Taherimehr, "Challenges in the catalytic synthesis of cyclic and polymeric carbonates from epoxides and $\mathrm{CO}_{2}, "$ Catalysis Science \& Technology, vol. 2, pp. 2169-2187, Jun. 2012. DOI: 10.1039/C2CY20365K.

[12] O. Hauenstein, S. Agarwal, A. Greiner, "Bio-based polycarbonate as synthetic toolbox," Nature Communications, vol.7, Article number: 11862, Jun. 2016. DOI: $10.1038 /$ ncomms 11862 .

[13] S. Klaus, M. W. Lehenmeier, C. E. Anderson, and B. Rieger, "Recent advances in $\mathrm{CO}_{2}$ /epoxide copolymerization-New strategies and cooperative mechanisms," Coordination Chemistry Reviews, vol. 255, pp. 1460-1479, Jul. 2011. DOI: 10.1016/j.ccr.2010.12.002.

[14] M. North, R. Pasquale, and C. Young, "Synthesis of cyclic carbonates from epoxides and $\mathrm{CO}_{2}$," Green Chemistry, vol. 12, pp. 1514-1539, Jun. 2010. DOI: 10.1039/C0GC00065E.

[15] S. D. Allen, D. R. Moore, E. B. Lobkovsky, and G. W. Coates, "High-Activity, Single-Site Catalysts for the Alternating Copolymerization of $\mathrm{CO}_{2}$ and Propylene Oxide," Journal of the American Chemical Society, vol. 124, pp. 14284-14285, Nov. 2002. DOI: 10.1021/ja028071g.

[16] M. Cheng, E. B. Lobkovsky, and G. W. Coates, "Catalytic Reactions Involving C1 Feedstocks: New High-Activity $\mathrm{Zn}$ (II)-Based Catalysts for the Alternating Copolymerization of Carbon Dioxide and Epoxides," Journal of the American Chemical Society, vol. 120, pp. 11018-11019, Oct. 1998. DOI: $10.1021 / \mathrm{ja} 982601 \mathrm{k}$.

[17] C. T. Cohen, C. M. Thomas, K. L. Peretti, E. B. Lobkovsky, and G. W. Coates, "Copolymerization of cyclohexene oxide and carbon dioxide using (salen)Co(iii) complexes: synthesis and characterization of syndiotactic poly(cyclohexene carbonate)," Dalton Transactions, pp. 237-249, Nov. 2006. DOI: 10.1039/B513107C.

[18] D. J. Darensbourg, R. M. Mackiewicz, A. L. Phelps, and D. R. Billodeaux, "Copolymerization of $\mathrm{CO}_{2}$ and Epoxides Catalyzed by Metal Salen Complexes," Accounts of Chemical Research, vol. 37, pp. 836-844, Nov. 2004. DOI: 10.1021/ar030240u.

[19] D. R. Moore, M. Cheng, E. B. Lobkovsky, and G. W. Coates, "Electronic and Steric Effects on Catalysts for $\mathrm{CO}_{2} /$ Epoxide Polymerization: Subtle Modifications Resulting in Superior Activities," Angewandte Chemie International Edition, vol. 41, pp. 2599-2602, Jul. 2002. DOI: 10.1002/15213773(20020715)41:14<2599::AID-ANIE2599>3.0.CO;2N.

[20] C. T. Cohen, T. Chu, and G. W. Coates, "Cobalt Catalysts for the Alternating Copolymerization of Propylene Oxide and Carbon Dioxide: Combining High Activity and Selectivity," Journal of the American Chemical Society, vol. 127, pp. 10869-10878, Aug. 2005. DOI: 10.1021/ja0517441.

[21] C. T. Cohen and G. W. Coates, "Alternating copolymerization of propylene oxide and carbon dioxide with highly efficient and selective (salen)Co(III) catalysts: Effect of ligand and cocatalyst variation," Journal of Polymer Science Part A: Polymer Chemistry, vol. 44, pp. 5182-5191, Jul. 2006. DOI: 10.1021/ma047551k.

[22] D. J. Darensbourg and S. J. Wilson, "What's new with $\mathrm{CO}_{2}$ ? Recent advances in its copolymerization with oxiranes," Green Chemistry, vol. 14, pp. 2665-2671, Jul. 2012. DOI: 10.1039/C2GC35928F.

[23] R. K. Dean, K. Devaine-Pressing, L. N. Dawe, and C. M. Kozak, "Reaction of $\mathrm{CO}_{2}$ with propylene oxide and styrene oxide catalyzed by a chromium(iii) amine-bis(phenolate) complex," Dalton Transactions, vol. 42, pp. 9233-9244, Jan. 2013. DOI: $10.1039 / \mathrm{c} 2 \mathrm{dt} 31942$ j.

[24] Z. Qin, C. M. Thomas, S. Lee, and G. W. Coates, "CobaltBased Complexes for the Copolymerization of Propylene Oxide and $\mathrm{CO}_{2}$ : Active and Selective Catalysts for Polycarbonate Synthesis," Angewandte Chemie International Edition, vol. 42, pp. 5484-5487, Nov. 2003. DOI: 10.1002/anie.200352605.

[25] W. Clegg, R. W. Harrington, M. North, and R. Pasquale, "Cyclic Carbonate Synthesis Catalysed by Bimetallic Aluminium-Salen Complexes," Chemistry - A European Journal, vol. 16, pp. 6828-6843, Apr. 2010. DOI: 10.1002/chem.201000030.

[26] K. Qiao, F. Ono, Q. Bao, D. Tomida, and C. Yokoyama, "Efficient synthesis of styrene carbonate from $\mathrm{CO}_{2}$ and styrene oxide using zinc catalysts immobilized on soluble imidazolium-styrene copolymers," Journal of Molecular Catalysis A: Chemical, vol. 303, pp. 30-34, Apr. 2009. DOI: 10.1016/j.molcata.2008.12.025.

[27] J. Sun, S.-i. Fujita, F. Zhao, and M. Arai, "Synthesis of styrene carbonate from styrene oxide and carbon dioxide in the presence of zinc bromide and ionic liquid under mild conditions," Green Chemistry, vol. 6, pp. 613-616, Oct. 2004. DOI: $10.1039 / \mathrm{B} 413229 \mathrm{G}$.

[28] G.-P. Wu, S.-H. Wei, X.-B. Lu, W.-M. Ren, and D. J. Darensbourg, "Highly Selective Synthesis of $\mathrm{CO}_{2}$ Copolymer from Styrene Oxide," Macromolecules, vol. 43, pp. 9202-9204, Nov. 2010. DOI: 10.1021/ma1021456. G.-P. Wu, S.-H. Wei, W.-M. Ren, X.-B. Lu, B. Li, Y.-P. Zu, et al., "Alternating copolymerization of $\mathrm{CO}_{2}$ and styrene oxide with $\mathrm{Co}$ (iii)-based catalyst systems: differences between styrene oxide and propylene oxide," Energy \& Environmental Science, vol. 4, pp. 5084-5092, Oct. 2011. DOI: $10.1039 / \mathrm{C} 1 \mathrm{EE} 02566 \mathrm{~J}$.

[30] C. M. Byrne, S. D. Allen, E. B. Lobkovsky, and G. W. Coates, "Alternating Copolymerization of Limonene Oxide and Carbon Dioxide," Journal of the American Chemical Society, vol. 126, pp. 11404-11405, Sept. 2004. DOI: $10.1021 / \mathrm{ja} 0472580$.

[31] W. Guo, V. Laserna, E. Martin, E. C. Escudero-Adán, and A. W. Kleij, "Stereodivergent Carbamate Synthesis by Selective in Situ Trapping of Organic Carbonate Intermediates," Chemistry - A European Journal, vol. 22, pp. 1722-1727, Jan. 2016. DOI: 10.1002/chem.201504510.

[32] C. Martín and A. W. Kleij, "Terpolymers Derived from Limonene Oxide and Carbon Dioxide: Access to CrossLinked Polycarbonates with Improved Thermal Properties," Macromolecules, vol. 49, pp. 6285-6295, Sept. 2016. DOI: 10.1021/acs.macromol.6b01449. 
[33] L. Peña Carrodeguas, J. González-Fabra, F. Castro-Gómez, C. Bo, and A. W. Kleij, "AlIII-Catalysed Formation of Poly(limonene)carbonate: DFT Analysis of the Origin of Stereoregularity," Chemistry - A European Journal, vol. 21, pp. 6115-6122, Apr. 2015. DOI: 10.1002/chem.201406334.

[34] J. Bailer, S. Feth, F. Bretschneider, S. Rosenfeldt, M. Drechsler, V. Abetz, H. Schmalz, A. Greiner, "Synthesis and self-assembly of biobased poly(limonene carbonate)-: Block -poly(cyclohexene carbonate) diblock copolymers prepared by sequential ring-opening copolymerization", Green Chemistry, vol. 21, no. 9, pp. 2266-2272, Apr. 2019. DOI: 10.1039/C9GC00250B.

[35] F. Parrino, A. Fidalgo, L. Palmisano, L.M. Ilharco, M. Pagliaro, R. Ciriminna, "Polymers of Limonene Oxide and Carbon Dioxide: Polycarbonates of the Solar Economy", ACS Omega, vol. 3, no. 5, pp. 4884-4890. May. 2018. DOI: 10.1021/acsomega.8b00644.

[36] A. Decortes, A. M. Castilla, and A. W. Kleij, "SalenComplex-Mediated Formation of Cyclic Carbonates by Cycloaddition of $\mathrm{CO}_{2}$ to Epoxides," Angewandte Chemie International Edition, vol. 49, pp. 9822-9837, Oct. 2010. DOI: 10.1002/anie.201002087.

[37] J. Rintjema, L. Peña Carrodeguas, V. Laserna, S. Sopeña, and A. W. Kleij, "Metal Complexes Catalyzed Cyclization with $\mathrm{CO}_{2}$," in Carbon Dioxide and Organometallics, X.-B. Lu, Ed., ed Cham: Springer International Publishing, Apr. 2016, pp. 39-71. DOI: 10.1007/3418_2015_94.

[38] S. J. Wezenberg and A. W. Kleij, "Material Applications for Salen Frameworks," Angewandte Chemie International Edition, vol. 47, pp. 2354-2364, Mar. 2008. DOI: 10.1002/anie.200702468.

[39] C. Martín, G. Fiorani, and A. W. Kleij, "Recent Advances in the Catalytic Preparation of Cyclic Organic Carbonates," ACS Catalysis, vol. 5, pp. 1353-1370, Feb. 2015. DOI: 10.1021/cs5018997.

[40] M. Taherimehr, A. Decortes, S. M. Al-Amsyar, W. Lueangchaichaweng, C. J. Whiteoak, E. C. Escudero-Adan, et al., "A highly active $\mathrm{Zn}$ (salphen) catalyst for production of organic carbonates in a green $\mathrm{CO}_{2}$ medium," Catalysis Science \& Technology, vol. 2, pp. 2231-2237, Apr. 2012. DOI: $10.1039 / \mathrm{C} 2 \mathrm{CY} 20171 \mathrm{~B}$.

[41] A. W. Kleij, M. Kuil, D. M. Tooke, M. Lutz, A. L. Spek, and J. N. H. Reek, "ZnII-Salphen Complexes as Versatile Building Blocks for the Construction of Supramolecular Box Assemblies," Chemistry - A European Journal, vol. 11, pp. 4743-4750, Jul. 2005. DOI: 10.1002/chem.200500227.

[42] J. F. Larrow, E. N. Jacobsen, Y. Gao, Y. Hong, X. Nie, and C. M. Zepp, "A Practical Method for the Large-Scale Preparation of [N,N'$\mathrm{Bis}$ (3,5-di-tertbutylsalicylidene)-1,2-cyclohexanediaminato(2)]manganese(III) chloride, a Highly Enantioselective Epoxidation Catalyst," The Journal of Organic Chemistry, vol. 59, pp. 19391942, Apr. 1994. DOI: 10.1021/jo00086a062.

[43] C. Martín and A. W. Kleij, "Comparing kinetic profiles between bifunctional and binary type of $\mathrm{Zn}$ (salen)-based catalysts for organic carbonate formation," Beilstein Journal of Organic Chemistry, vol. 10, pp. 1817-1825, Aug. 2014. DOI: 10.3762/bjoc.10.191.

[44] D. R. Moore, M. Cheng, E. B. Lobkovsky, and G. W. Coates, "Mechanism of the Alternating Copolymerization of Epoxides and $\mathrm{CO}_{2}$ Using $\beta$-Diiminate Zinc Catalysts: Evidence for a Bimetallic Epoxide Enchainment," Journal of the American Chemical Society, vol. 125, pp. 11911-11924, Oct. 2003. DOI: 10.1021/ja030085e.

[45] A. Decortes and A. W. Kleij, "Ambient Fixation of Carbon Dioxide using a ZnIIsalphen Catalyst," ChemCatChem, vol. 3, pp. 831-834, Mar. 2011. DOI: 10.1002/cctc.201100031.

[46] A. Decortes, M. Martinez Belmonte, J. Benet-Buchholz, and A. W. Kleij, "Efficient carbonate synthesis under mild conditions through cycloaddition of carbon dioxide to oxiranes using a $\mathrm{Zn}$ (salphen) catalyst," Chemical Communications, vol. 46, pp. 4580-4582, May. 2010. DOI: $10.1039 /$ C000493F.

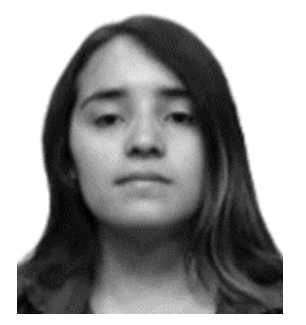

Sherly Catherin Acosta Beltrán nació en Bogotá, Colombia. Obtuvo su título de Química en septiembre de 2017. Desarrolló su trabajo de grado bajo la dirección de la profesora Carolina Blanco en el cual estudió la síntesis y la caracterización de catalizadores homogéneos para la copolimerización de epóxidos y dióxido de carbono. Los intereses en la investigación que tiene la Química Acosta están orientados hacia la síntesis de nuevos compuestos de coordinación con potencial uso como catalizadores para reacciones que permitan la mitigación y uso de gases de efecto invernadero.

ORCID: https://orcid.org/0000-0002-2007-9356

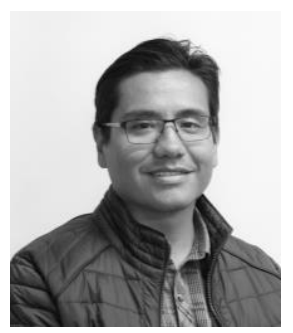

Carlos Daza nació en Bogotá, Colombia. Es Químico de la Universidad Nacional de Colombia (2004) y Doctor en Ciencias-Química de la misma institución (2009). Su tesis de doctorado (mención Laureada) fue desarrollada en el área de la Catálisis Heterogénea Ambiental. El Dr. Daza pertenece al grupo Estado Sólido y Catálisis Ambiental de la Universidad Nacional de Colombia y por su investigación está reconocido por COLCIENCIAS como investigador senior desde 2016. Sus intereses investigativos están enfocados en el desarrollo de catalizadores sólidos de bajo costo para reacciones con impacto positivo en el medio ambiente. Actualmente, el Dr. Daza es Profesor Asociado del Departamento de Química de la Universidad Nacional de Colombia.

ORCID: https://orcid.org/0000-0002-9521-9499

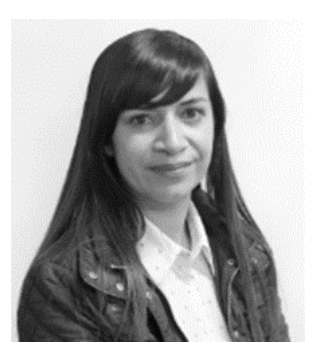

Carolina Blanco Jiménez nació en Bogotá, Colombia. Obtuvo su título de Química, en el año 2005, en la Universidad Nacional de Colombia. En el año 2010, obtuvo su título de Doctora en Química (mención sobresaliente "cum laude") en la Universitat Rovira i Virgili. Su investigación se centró en los procesos de carbonilación asimétrica de alquenos catalizada por metales de transición. En el año 2010, se vinculó como investigadora post-doctoral en el Instituto Catalán de Investigación Química y bajo la supervisión del Prof. Piet Van Leeuwen, trabajó en un proyecto industrial con la compañía suiza Firmenich. La Dra. Blanco tiene amplia experiencia en el campo de la catálisis homogénea, actualmente tiene un profundo interés en la mejora del impacto ambiental de los procesos catalíticos a través del estudio de los 
aspectos de reducción de residuos, utilización de disolventes alternativos, uso de metales de baja toxicidad, etc., conservando siempre la máxima selectividad de las reacciones químicas. Actualmente, la Dra. Blanco es profesora Asistente del Departamento de Química de la Universidad Nacional de Colombia.

ORCID: http://orcid.org/0000-0003-2659-7805 